https://doi.org/10.46344/JBINO.2021.v10i02.27

\title{
SODIUM VALPROATE-RELATED PERIPHERAL OEDEMA IN A PATIENT OF BIPOLAR AFFECTIVE DISORDER: A CASE REPORT
}

\author{
Dr. Chayanika Bharadwaj', Dr. Kunal Deb², Dr. Nishant Saha³ , Dr. Ramling Dhabale ${ }^{4}$ \\ 1 PGT third year, ${ }^{2}$ Assistant Professor, ${ }^{3}$ PGT third year, 4 Senior Resident, LGBRIMH, Tezpur, Assam, India \\ (Received on Date: 17 March 2021 \\ Date of Acceptance: 18 March 2021 \\ Date of Publish: 19 March 2021) \\ email id- fantasy.1123@yahoo.in
}

\begin{abstract}
Peripheral oedema is a common clinical complaint with a heterogenous etiological basis. Although drug related etiologies are not rare, they are also not a common occurrence with respect to psychiatric medications. Sodium Valproate is a drug with a broad array of indications in epilepsy, bipolar disorder, schizoaffective disorder and prophylaxis of migraine. Bipolar affective disorder is characterized by episodes in which the the patient's mood and activity levels are significantly disturbed, at times presenting with elevation of mood and energy and at times with lowering of it. This report describes the manifestation of peripheral oedema in a patient with Bipolar disorder after starting valproate in the management. It underlies the importance of weight and oedema monitoring in all patients on sodium valproate, early identification of peripheral oedema and targeted management.
\end{abstract}

Keywords: Oedema, Drug, Valproate, Bipolar disorder 


\section{INTRODUCTION}

Peripheral oedema is a common clinical complaint with a heterogenous etiological basis. It is usually a manifestation of an increased interstitial volume in limbs bilaterally. Many causes of peripheral edema should be considered such as hypoalbuminemia, cirrhosis, nephrotic syndrome, hypothyroidism, or congestive heart failure. Although drug related etiologies are not rare, they are also not a common occurrence with respect to psychiatric medications.

Bipolar affective disorder is characterized by episodes in which the the patient's mood and activity levels are significantly disturbed, at times presenting with elevation of mood and energy and at times with lowering of it.

Sodium Valproate is a drug with a broad array of indications in epilepsy, bipolar disorder, schizoaffective disorder and prophylaxis of migraine.. Although long term usage of valproate related peripheral oedema has been documented infrequently, scarcity of reports has partially impeded proper understanding and delineation of the mechanisms underlying it.

\section{CASE DESCRIPTION}

Mr. $\mathrm{H}, \quad \mathrm{a} 45$ years old gentleman presented in OPD with the complaints of decreased sleep, increased talkativeness with boastfulness, singing and dancing behavior, overfamiliarity and over religiosity. On mental status examination, patient's approach was overfamiliar and boisterous, speech showed increased productivity with decreased reaction time, affect was euphoric, and thought content showed delusion of grandiosity and religious preoccupation, judgement

was impaired and insight Grade 2. These symptoms had evolved acutely over a period of 2 weeks. Past history revealed presence of similar symptoms in an episodic pattern over a total duration of 25 years. Altogether there were 9 such episodes in the past.

A provisional diagnosis of Bipolar affective disorder, current episode mania with psychotic symptoms was made.

Reviewing the treatment history, it showed that the earlier episodes had remitted on a regimen of Tab Haloperidol $15 \mathrm{mg}$, Tab Carbamazepine, $800 \mathrm{mg}$, Tab Trihexyphenidyl, $2 \mathrm{mg}$. Following a relapse of symptoms in 2016, even on medications, patient was put on Tab Olanzapine, $15 \mathrm{mg}$ and Tab Sodium Valproate, $600 \mathrm{mg}$ and patient remitted on these medications. Patient was maintaining on these medications till March 2020, when there was irregularity in compliance for a period of 2 months following which symptoms exacerbated. Tab Olanzapine $10 \mathrm{mg}$ was started again, uptitrated to $20 \mathrm{mg}$ over 2 weeks, and Sodium Valproate, $400 \mathrm{mg}$ uptitrated to $600 \mathrm{mg}$ in 4 days, and then to $800 \mathrm{mg}$ in another 5 days, along with Trihexyphenidyl,2mg. The plan was to uptitrate to $1000 \mathrm{mg}$, after obtaining the serum valproate levels.

Around 2 weeks after uptitrating valproate to $800 \mathrm{mg}$, patient started developing peripheral pitting oedema. It started initially in bilateral feet, and proceeded to involve the lower legs and also the dorsum of hands.

Systemic examination revealed no abnormal findings. Laboratory results of 
complete blood cell count, electrolytes, blood urea nitrogen, serum creatinine, liver enzymes, albumin, free thyroxine, free triiodothyronine, thyroid stimulating hormone were within normal limits. Chest radiographs, ECG, USG(W/A) were within normal limits. Serum valproate level was $53 \mathrm{ugm} / \mathrm{ml}$.

Suspecting a drug related etiology, Sodium valproate was reduced to 600 mg, within 1 week there was improvement in the condition, although oedema around the ankles persisted. As the manic symptoms were better controlled, the drug regimen was continued and patient was discharged on these medications.

In the follow up in May 2020, patient had complaints of excessive sedation, and considering it was affecting compliance, olanzapine was stopped and risperidone $6 \mathrm{mg}$ was started. As pitting oedema was still persisting in bilateral feet, sodium valproate was tapered down and lithium $600 \mathrm{mg}$ was started in the follow up in August 2020. Within 5 days of stopping Sodium Valproate, there was complete subsidence of peripheral oedema.

\section{DISCUSSION}

After excluding other causes, the temporal course of events described suggest an association between use of valproate and the development of peripheral oedema. Indeed, oedema related to risperidone also needs to be considered but was considered less likely in this case as oedema subsided following stopping valproate even when risperidone was continued, suggesting probable causality related to valproate in Naranjo adverse drug reaction probability scale.
Most antecedent reports has shown valproate related peripheral oedema in long term administration (mean duration 3.9 years) of high doses (>1500 mg) in cases of epilepsy in children as well as adults (Ettinger 1990). However, instances of acute onset of oedema within days of initiating low doses of valproate 1500 mg$800 \mathrm{mg}$ ) in bipolar affective disorder and subsidence of oedema following its cessation have also been documented (Maneerot 2019; Panchal 2017).

The mechanisms are still unclear. Longterm or high-dose valproate therapy may elicit a greater degree of cytochrome P450-mediated omega oxidation, increasing the risk of the dose related and idiosyncratic hepatic and metabolic adverse effects of valproate (Anderson \& Ritland, 1995). Valproate up-regulates NADPH oxidase, COX-2, ERK $1 / 2$ and HIF- 1 (Hypoxia Inducible Factor), (NOX), generator of ROS resulting in an increase in oxidative stress, hypoxia, inflammation and apoptosis, aggravation of inflammation, mitogenesis, fibrosis, steatosis.

Other mechanisms are related to the nonspecific calcium channel inhibitor property can cause peripheral edema similar to dihydropyridine calcium channel blockers. Moreover, it was found that the drug class gabapentinoids including gabapentin and pregabalin, a $P / Q$-type calcium channel inhibitor, a drug for neuropathic pain, can cause peripheral edema and similar mechanisms have been cited for valproate.

\section{CONCLUSION}

It implies the importance of weight and oedema monitoring in all patients on 
sodium valproate, early identification of peripheral oedema and targeted management.

\section{REFERENCES}

Andersen, G. O., \& Ritland, S. (1995). Life threatening intoxication with sodium valproate. Journal of toxicology. Clinical toxicology, 33(3), 279-284. https://doi.org/10.3109/1556365950901800 0

Ettinger, A., Moshe, S., \& Shinnar, S. (1990). Edema associated with long-term valproate therapy. Epilepsia, $31(2), 211-$ 213. https://doi.org/10.1111/j.15281167.1990.tb06308.x

LiverTox: Clinical and Research Information on Drug-Induced Liver Injury [Internet]. Bethesda (MD): National Institute of Diabetes and Digestive and Kidney Diseases; 2012-. Adverse Drug Reaction Probability Scale (Naranjo) in Drug Induced Liver Injury. [Updated 2019 May 4]. Available from: https://www.ncbi.nlm.nih.gov/books/NBK 548069/

Maneerot, T., Jenraumiit, R., Kijsawatpaiboon, S., \& Reungyos, J. (2019). Low-Dose Valproate-Related Peripheral Edema in Patients With Bipolar Disorder: Two Case Reports. Journal of clinical psychopharmacology, 39(4), 412413.

Panchal, R., Chaudhary, D., \& Anovadiya, A. (2018). Sodium ValproateInduced Bilateral Pitting Pedal Edema - A Case Report. Current drug safety, 13(2), 128-130. https://doi.org/10.2174/15748866312666170 804164854 